\title{
Ebola virus and persistent chronic infection: when does replication cease?
}

\author{
John V. Forrester ${ }^{1,2}$ \\ ${ }^{1}$ Institute of Medical Sciences, University of Aberdeen, Aberdeen, UK; ${ }^{2}$ Lions Eye Institute, University of Western Australia, Perth, Western \\ Australia, Australia \\ Correspondence to: John V. Forrester. Institute of Medical Sciences, University of Aberdeen, Aberdeen, UK; University of Western Australia, Lions \\ Eye Institute, Perth, Western Australia, Australia. Email: j.forrester@abdn.ac.uk. \\ Comment on: Shantha JG, Mattia JG, Goba A, et al. Ebola Virus Persistence in Ocular Tissues and Fluids (EVICT) Study: Reverse Transcription- \\ Polymerase Chain Reaction and Cataract Surgery Outcomes of Ebola Survivors in Sierra Leone. EBioMedicine 2018;30:217-24.
}

Submitted Aug 27, 2018. Accepted for publication Sep 20, 2018.

doi: $10.21037 / \mathrm{atm} .2018 .09 .60$

View this article at: http://dx.doi.org/10.21037/atm.2018.09.60

In this issue of the journal, Shantha et al. report a series of cataract surgeries on survivors of Ebola virus (EBOV) infection. EBOV, originally identified as a new pathogen in 1976 during an outbreak of haemorrhagic fever in Zaire (1), is a highly virulent organism associated with a mortality rate ranging from 50 to nearly $90 \%$ [reviewed in (2)]. Up to $40 \%$ of EBOV survivors develop recurrent inflammation at certain sites (eye, testis, synovium, meninges) despite being tested virus-negative. These are sites of attenuated immunity (so-called "immune privilege"), a property which is dependent on the nature of the tissue environment (3). During recurrence of disease (uveitis, synovitis, arthritis, etc.), EBOV RNA is commonly detected in the extracellular compartment and EBOV can be readily cultured from tissue samples in these sites (4), suggesting that persistent or latent infection is the cause of the recurrent inflammation. In the case of EBOV disease (EBOVD), recurrence is presumed to be due to chronic persistent infection rather than reactivation of latent infection as occurs with DNA viruses such as cytomegalovirus (CMV) (5). In chronic persistent infection, disease is caused by an actively replicating virus, but presumably at low level. In contrast, in latent infection replication has ceased, although the virus remains replication competent. This distinction is important since transmission of EBOVD occurs through direct contact, and thus the presence of replicating virus, in conjunction with the virus' duration, has implications for infectivity, for instance in the case of health workers treating EBOV survivors. Specifically, when can the virus-negative EBOVD survivor be considered not to be a health risk?
Much has been learned in recent years concerning the biology of EBOV. EBOV, is an anti-sense ssRNA filovirus, which along with Marburg-virus and Cuevavirus are classed as agents which cause haemorrhagic fever (2) and are thought to act through similar pathogenetic mechanisms. There are five known species of EBOV which are identified by the country where the first outbreak occurred [reviewed in (6)]. Four of these species are pathogenic to humans. Infection is contracted by exposure to infected animal tissue, by human-to-human fluid contact or by vertical transmission [reviewed in (7)]. Viral entry is thought to occur through skin abrasions or through mucosal surfaces.

EBOV induces an exaggerated innate immune response. Infection of several cell types occurs but tissue myeloid cells are primary targets with later spread to other cells such as fibroblasts, epithelia and muscle cells. Viral entry into myeloid cells occurs via a range of host receptors such as dendritic cell-specific intercellular adhesion molecule specific-3 integrin (DC-SIGN) (8), TAM-receptor tyrosine kinases $(9,10)$, and $\mathrm{T}$ cell immunoglobulins and mucin domain-1 protein (TIM-1) (11). These receptors, especially DC-SIGN and TIM-1 are present on myeloid cells while TIM-1 has broader expression on other cells such as B and $\mathrm{T}$ cells and can contribute to non-specific $\mathrm{T}$ cell activation. Infection of this range of cells, particularly macrophages and neutrophils, leads to massive cytokine and chemokine production, including TNF $\alpha$, IL-1, IL-6, IL-8, IL-15, IL-16, CCLs-2, -3 and -4, CXCL-10, MCSF and macrophage migration inhibition factor. This is in part mediated via TLR4 activation as well as signalling through the triggering 
receptor of myeloid cells (TREM) which sends neutrophils into overdrive adding to the cytokine production (12). There is also an outpouring of reactive oxygen and nitrogen species as well as Tissue Factor and other pro-coagulant proteins.

Control of viral replication by the immune system is also impaired. Inhibition of Type 1 interferon production by blockade of IRF3 signalling is mediated by Ebola viral protein 35 (VP35), which prevents adequate viral clearance, allowing free rein to viral replication. This amplifies the cytokine/chemokine response as more mononuclear phagocytes are recruited to sites of inflammation with further cytokine production. The combined cytokine storm and disseminated intravascular coagulopathy (DIC) leads to hypovolemic shock, hypotension, and death (7). Much of this pathology is mediated by the EBOV surface protein GP, which is shed in large amounts from the infected cell surface, as well as being released as soluble protein (sGP) from live and dying macrophages (13). GP is the major mediator of myeloid cell infection via MerTK (14) and is a potent activator of macrophages.

Concomitant targeting of dendritic cells has profound effects on the adaptive immune response to EBOV. VP35 in addition to blocking type 1 interferon production, also inhibits the maturation of dendritic cells and thus prevents an adequate $T$ cell response required to kill virus-infected cells (15). Infected immature dendritic cells release viral matrix protein (VP40)-containing exosomes which are delivered to naive $\mathrm{T}$ cells (16) and induce apoptosis. Nonactivated CD4 and $8 \mathrm{~T}$ cells also undergo apoptosis, mediated by macrophages, through several mechanisms including upregulated expression of TRAIL (17). Collectively, this process accounts for the severe lymphopenia in these patients.

Survival depends on how well the innate and adaptive immune responses can contain viral proliferation. As indicated above, EBOV infection of dendritic cells is the likely cause of the poor adaptive immune response and, unless infected cells can be cleared from the system by a sufficient $\mathrm{T}$ cell response, the outcome is poor. Individuals who have survived have clearly generated a sufficient adaptive response to clear the virus from vital organs but recurrence of infection as a late complication indicates either viral persistence or reactivation. Reservoirs of infection could implicate several cells types but myeloid cells are a strong contender for this role. Resident tissue macrophages and dendritic cells are recruited from the adult bone marrow, a tissue which is heavily infected during the acute phase of infection, as evidenced by the numerous inclusion bodies contained within bone marrow cells and the extensive deposition of extracellular viral antigen in the bone marrow stroma (18). Since bone marrow cells continue to be produced throughout life, it is likely, if not inevitable, that myeloid-dendritic progenitor (MDP) cells also become infected with EBOV. MDP cells in their normal life cycle continue to enter the blood circulation and populate the tissues where they differentiate to become resident tissue macrophages and dendritic cells. If infected, such cells could act as reservoirs of viral persistence in the tissues at least for the lifetime of the cell, which may be several weeks to months if not longer. Interestingly, EBOV+ CD68+ monocytic cells have recently been detected in the vitreous in a primate model of Ebola infection (19). A myeloid cell niche has been proposed as a common mechanism for viral persistence in other infections such as Dengue, Chikungunya and Marburg virus (7). In the case of DNA viruses such as CMV, viral targeting of myeloid cells is a prominent mechanism for achieving latent infection (20).

This brings back the important question of how EBOV establishes a chronic persistent infection and whether this slowly replicating virus is in replicative decline. In EBOVD, infected dendritic cells, which traffic from the bone marrow as progenitors, and reside in the secondary lymphoid and non-lymphoid tissues, are likely to be surveyed by $\mathrm{T}$ and $\mathrm{B}$ cells, albeit as part of a struggling adaptive immune response in the case of Ebola survivors. In tissues where there are sufficient virus specific $\mathrm{T}$ cells to respond to and remove the infected cells, the virus will be cleared. However, in tissues such as the uveal tract, the meninges, the synovium and the testis, where the infected myeloid cell has limited exposure to memory $\mathrm{T}$ cells, the newly recruited infected dendritic cell may survive and permit the virus to proliferate unchecked or partially checked (low grade chronic inflammation). This might explain the random recurrence of disease in these tissues, since the balance between low level immune cell surveillance and low level viral replication will be constantly shifting. In addition, if viral replication in these sites gains the upper hand and expands, it will threaten the organism by spreading to other organs and tissues.

The above mechanism thus might explain the recurrence of disease in EBOV survivors in sites such as the eye, joint or testis and probably is an indicator of a partially effective systemic memory $\mathrm{T}$ cell response. This response is probably sufficiently strong to allow adequate recruitment of cytotoxic cells to the site to clear the virus and, in the 
process, manifests clinically as a prominent/severe uveitis or enthesitis (etc.). The payoff however depends on the tissue and includes for instance, severe damage to the eye and loss of vision, or severe testicular damage and infertility. In the eye, lower levels of inflammation are manifest as secondary complications such as cataract and the hypotony of low grade cyclitis (21). The ocular damage is as likely to be caused by the immune response as by direct viral cytotoxicity and indeed this notion is borne out by the partial response of infected sites to immunosuppressive drugs. However, the beneficial effect of antiviral drug favipiravir in the case of uveitis suggests that persistent viral replication is also a factor in tissue damage (4).

The paper by Shantha et al. addresses the issue of the reservoir of EBOV in the eye and by implication the lifespan of EBOV-infected cells. The study (the EVICT Study) was designed to prospectively determine whether cataract surgery might lead to a resurgence of uveitis in patients who had already suffered EBOV-induced uveitis. It is believed by many that various stimuli, including physical trauma, may exacerbate or even amplify a low grade persistent infection. As indicated above, in survivors of EBOV, it is likely that the virus resides in ciliary body or iris myeloid cells which constitute a dense network of cells in the healthy eye and which are constantly replenished from bone marrow progenitors (22). The replication cycle of persistent slowlyreplicating virus in such cells would likely be accelerated by the trauma of cataract surgery if only by release of virions to infect neighbouring cells. However, Shantha et al. report that there were no recurrences of viral replication or of uveitis in any of survivors of EBOVD who underwent cataract surgery when the eye was uninflamed. Surgery was not undertaken in cases where inflammation was active since viral activity was assumed. The failure to reactivate viral infection in this study suggests that recurrence of disease in EBOV survivors is the result of persistent low grade, replicating virus, which escapes immune control in certain tissues such as the eye or testis but can eventually be cleared. A recent study in primates supports this notion (19).

While these data encourage the belief that permanent clearance of virus has occurred after it becomes undetectable symptomatically and by repeated sampling in survivors of Ebola, caution is advised until further evidence is gathered, not least because of the extreme consequences of full-blown Ebola infection. In addition, the duration of a viral-free period and the necessary frequency of sampling fluids and tissues before the all clear can be given are not known and in some cases might be very long. The intervals from the original EBOVD in the series reported by Shantha et al. were 19 months in the phase 1 cohort and 34 months in the phase 2 cohort of patients. More positively, analysis of persistent virus in semen, urine and aqueous samples suggests that persistent EBOV is in a low level replicative phase, and is not a dormant or latent infection (23). The data from Shantha et al. would support this contention. However, if the above pathogenesis of EBOV recurrence is correct, then the bone marrow would seem to be the source of infected cells and a clean bill of health for survivors of EBOV might require evidence of viral-free bone marrow.

\section{Acknowledgements}

Supported by Saving Sight in Grampian and the Development Trust of the University of Aberdeen.

\section{Footnote}

Conflicts of Interest: The author has no conflicts of interest to declare.

\section{References}

1. Johnson KM, Lange JV, Webb PA, et al. Isolation and partial characterisation of a new virus causing acute haemorrhagic fever in Zaire. Lancet 1977;1:569-71.

2. Cantoni D, Rossman JS. Ebolaviruses: New roles for old proteins. PLoS Negl Trop Dis 2018;12:e0006349.

3. Matzinger P, Kamala T. Tissue-based class control: the other side of tolerance. Nat Rev Immunol 2011;11:221-30.

4. Varkey JB, Shantha JG, Crozier I, et al. Persistence of Ebola Virus in Ocular Fluid during Convalescence. N Engl J Med 2015;372:2423-7.

5. Goodrum F. Human Cytomegalovirus Latency: Approaching the Gordian Knot. Annu Rev Virol 2016;3:333-57.

6. Rivera A, Messaoudi I. Molecular mechanisms of Ebola pathogenesis. J Leukoc Biol 2016;100:889-904.

7. Supramaniam A, Lui H, Bellette BM, et al. How myeloid cells contribute to the pathogenesis of prominent emerging zoonotic diseases. J Gen Virol 2018;99:953-69.

8. Alvarez CP, Lasala F, Carrillo J, et al. C-type lectins DCSIGN and L-SIGN mediate cellular entry by Ebola virus in cis and in trans. J Virol 2002;76:6841-4.

9. Shimojima M, Takada A, Ebihara H, et al. Tyro3 familymediated cell entry of Ebola and Marburg viruses. J Virol 2006;80:10109-16. 
10. Hunt CL, Kolokoltsov AA, Davey RA, et al. The Tyro3 receptor kinase Axl enhances macropinocytosis of Zaire ebolavirus. J Virol 2011;85:334-47.

11. Kondratowicz AS, Lennemann NJ, Sinn PL, et al. T-cell immunoglobulin and mucin domain 1 (TIM-1) is a receptor for Zaire Ebolavirus and Lake Victoria Marburgvirus. Proc Natl Acad Sci U S A 2011;108:8426-31.

12. Mohamadzadeh M, Coberley SS, Olinger GG, et al. Activation of triggering receptor expressed on myeloid cells-1 on human neutrophils by marburg and ebola viruses. J Virol 2006;80:7235-44.

13. Kawaoka Y. How Ebola virus infects cells. N Engl J Med 2005;352:2645-6.

14. Dahlmann F, Biedenkopf N, Babler A, et al. Analysis of Ebola Virus Entry Into Macrophages. J Infect Dis 2015;212 Suppl 2:S247-57.

15. Yen B, Mulder LC, Martinez O, et al. Molecular basis for ebolavirus VP35 suppression of human dendritic cell maturation. J Virol 2014;88:12500-10.

16. Pleet ML, Mathiesen A, DeMarino C, et al. Ebola VP40 in Exosomes Can Cause Immune Cell Dysfunction. Front Microbiol 2016;7:1765.

17. Gupta M, Spiropoulou C, Rollin PE. Ebola virus infection

Cite this article as: Forrester JV. Ebola virus and persistent chronic infection: when does replication cease? Ann Transl Med 2018;6(Suppl 1):S39. doi: 10.21037/atm.2018.09.60 of human PBMCs causes massive death of macrophages, CD4 and CD8 T cell sub-populations in vitro. Virology 2007;364:45-54.

18. Martines RB, Ng DL, Greer PW, et al. Tissue and cellular tropism, pathology and pathogenesis of Ebola and Marburg viruses. J Pathol 2015;235:153-74.

19. Zeng X, Blancett CD, Koistinen KA, et al. Identification and pathological characterization of persistent asymptomatic Ebola virus infection in rhesus monkeys. Nat Microbiol 2017;2:17113.

20. Voigt V, Andoniou CE, Schuster IS, et al. Cytomegalovirus establishes a latent reservoir and triggers long-lasting inflammation in the eye. PLoS Pathog 2018;14:e1007040.

21. Shantha JG, Crozier I, Yeh S. An update on ocular complications of Ebola virus disease. Curr Opin Ophthalmol 2017;28:600-6.

22. Forrester JV, Xu H, Kuffova L, et al. Dendritic cell physiology and function in the eye. Immunol Rev 2010;234:282-304.

23. Whitmer SLM, Ladner JT, Wiley MR, et al. Active Ebola Virus Replication and Heterogeneous Evolutionary Rates in EVD Survivors. Cell Rep 2018;22:1159-68. 